\title{
ON A NECESSARY AND SUFFICIENT CONDITION THAT AN INFINITELY DIVISIBLE DISTRIBUTION BE ABSOLUTELY CONTINUOUS $\left({ }^{1}\right)$
}

\author{
BY \\ HOWARD G. TUCKER
}

1. Introduction and summary. An infinitely divisible probability distribution function $F$ is a distribution function satisfying the property that for every positive integer $n$ there exists a distribution function $F_{n}$ such that the $n$-fold convolution, $F_{n}^{* n}$, of $F_{n}$ with itself is $F$, i.e., $F=F_{n} * \cdots * F_{n}$ ( $n$ times). Its characteristic function $f$ has a unique representation of the form

$$
f(u)=\exp \left\{i \gamma u+\int_{-\infty}^{\infty}\left(e^{i u x}-1-\frac{i u x}{1+x^{2}}\right) \frac{1+x^{2}}{x^{2}} d G(x)\right\},
$$

called the Lévy-Khinchin representation, where $\gamma$ is a real constant, and $G$ is a bounded, nondecreasing function. If one defines the function $M$ over $(-\infty, 0)$ $\cup(0, \infty)$ by

$$
M(x)= \begin{cases}\int_{-\infty}^{x}\left(\left(1+\tau^{2}\right) / \tau^{2}\right) d G(\tau) & \text { if } x<0, \\ -\int_{x}^{\infty}\left(\left(1+\tau^{2}\right) / \tau^{2}\right) d G(\tau) & \text { if } x>0,\end{cases}
$$

then another unique representation of $f$ is

$$
f(u)=\exp \left\{i \gamma u-\sigma^{2} u^{2} / 2+f_{-\infty}^{\infty}\left(e^{i u x}-1-\frac{i u x}{1+x^{2}}\right) d M(x)\right\},
$$

where $\sigma^{2}=G(+0)-G(1-0) \geqq 0$. Comprehensive developments of the theory of infinitely divisible distributions are contained in books by Gnedenko and Kolmogorov [4], Loève [7], and Lukacs [8].

It is easy to see that $M$ is absolutely continuous over $(-\infty, 0) \cup(0, \infty)$ if and only if $G$ is, and the same statement can be made for the properties: continuous singular and discrete. A problem that has been considered for some time in connection with infinitely divisible distributions is that of their Lebesgue properties

Presented to the Society, April 21, 1964; received by the editors February 6, 1964.

(1) This research was supported in part by the Air Force Office of Scientific Research, Grant No. AF-AFOSR-62-328 and National Science Foundation, Grant No. GF-1365. 
in terms of the $M$-functions (or in terms of their $G$-functions), i.e., properties of absolute continuity, continuous singularity and discreteness. The earliest such investigation is perhaps that of $P$. Hartman and A. Wintner [5]. In this paper they proved that a necessary and sufficient condition that $F$ be continuous is that $f_{-\infty}^{\infty} d M(x)=\infty$ or $\sigma^{2}>0$. They also gave a necessary condition that $F$ be absolutely continuous and a sufficient condition that $F$ be absolutely continuous. They could not find a condition that is both necessary and sufficient, and they remarked: "The difficulties of the gap between these two conditions appear to be of diophantine irregularity and are rather obscure" (p. 287 in [5]). The approach taken by them was through the use of the Riemann-Lebesgue lemma and other methods of Fourier analysis which, it now appears, are too crude for such a problem. The above investigation by Hartman and Wintner was not too generally known. In 1959, J. Blum and M. Rosenblatt [1] obtained the same necessary and sufficient condition that $F$ be continuous but with an entirely different proof. In a special invited paper [2] entitled Infinitely divisible distributions: recent results and applications, presented in 1961 to the Annual Meeting of the Institute of Mathematical Statistics, M. Fisz remarked (on p. 72): "As of now, no conditions are known for $F(x)$ to be absolutely continuous." Another sufficient condition soon appeared for absolute continuity of $F$, namely, that if $M_{a c}$ denotes the absolutely continuous component of $M$ in (1.2), and if $f_{-\infty}^{\infty} d M_{a c}(x)=\infty$, then $F$ is absolutely continuous. This result is contained in two almost simultaneously appearing papers, one by by M. Fisz and V. S. Varadarajan [3] and one by the author [10], the two proofs being somewhat different. Other Lebesgue properties of $F$ have received some attention. One of the interesting results in the paper by Hartman and Wintner referred to above is that if the $M$-function is discrete (i.e., generates a purely atomic Lebesgue-Stieltjes measure over $(-\infty, 0) \cup(0, \infty))$, then the distribution of $F$ is pure, and each of the three types is possible. (This result is used in the proof of Theorems 1 and 3 in this paper.) Sufficient conditions for a discrete $M$ to produce a continuous singular $F$ are given in [11]; these conditions are far from necessary and do not even contain the example given in [5] of a discrete $M$-function which produces a continuous singular $F$.

The purpose of this paper is to present a theorem which in an unsatisfactory, but perhaps best, way gives a condition on $M$ which is necessary and sufficient for $F$ to be absolutely continuous. In the remainder of this section we state this result, discuss its meaning and summarize the contents of this paper.

Let $M_{a c}, M_{c s}, M_{d}$ denote the absolutely continuous, the continuous singular and the discrete components, respectively, of $M$ in (1.2). Let $F^{(i)}$ denote the infinitely divisible distribution function produced by the $M$-function, $M_{i}$, where $i=a c, c s, d$. (Let $\gamma=0$ since it has nothing to do with the question being discussed.) The following theorem is proved in this paper.

THEOREM 1. Let $F$ be an infinitely divisible distribution function with charac- 
teristic function (1.2) (or (1.1)). A necessary and sufficient condition that $F$ be absolutely continuous is that at least one of the following five conditions hold:

(i) $f_{-\infty}^{\infty} d M_{a c}(x)=\infty$,

(ii) $\sigma^{2}>0$ (or $G$ is not continuous at 0$)$,

(iii) $F^{(d)}$ is absolutely continuous,

(iv) $F^{(c s)}$ is absolutely continuous, or

(v) $F^{(d)}$ is continuous singular, $F^{(c s)}$ is continuous but not absolutely continuous, and $F^{(d)} * F^{(c s)}$ (convolution) is absolutely continuous.

N. B. The above theorem does not state that each of conditions (i)-(v) is necessary, but it does state that at least one of them is necessary.

Each of conditions (ii)-(v) is obviously sufficient for absolute continuity of $F$. The sufficiency of (i) was proved in [3] and [10]. It is doubtful that any substantially better necessary and sufficient condition can be obtained, and for the following reason. In view of the theorem by Blum and Rosenblatt and by Hartman and Wintner given above for continuity of $F$, the best one could hope for is that $F$ is absolutely continuous if and only if $\phi(\varepsilon)=\int_{-1}^{-\varepsilon}+\int_{\varepsilon}^{1} d M(x)$ tends to infinity at a certain rate as $\varepsilon \rightarrow 0(\varepsilon>0)$. However, such a condition could not be necessary since $F$ could be absolutely continuous and this growth condition can be violated by simply having $f_{-\infty}^{\infty} d M_{a c}(x)=\infty$, where $M_{a c}$ violates this growth condition, and $M_{d}$ and $M_{c s}$ are missing.

In $\$ 2$ some lemmas and their proofs (or references to their proofs) are given. Also, the proof of Theorem 1 is given, and the possibility of occurrence of each of conditions (i), (ii), and (iii) is demonstrated. Actually, Theorem 1 does not say very much and is not of great interest. What appears to be of some interest is the possibility of fulfillment of conditions (iv) and (v) of Theorem 1; this requires the greatest effort, and it is accomplished in $\S \S 3$ and 4 . In $\S 3$ a very ingenious model of an infinitely divisible distribution $F_{1}$, due to Herman Rubin [9], is constructed which has the property that its $M$-function is discrete, $F_{1}$ is continuous singular and $F_{1} * F_{1}$ is absolutely continuous. In $\$ 4$, Rubin's model is altered, enabling us to construct an infinitely divisible distribution function $F_{2}$ whose $M$-function is continuous singular, such that $F_{2}$ is continuous but not absolutely continuous, and such that $F_{1} * F_{2}$ and $F_{2} * F_{2}$ are both absolutely continuous, thus demonstrating the nonvacuousness of conditions (iv) and (v).

2. Some lemmas and the proof of Theorem 1. Gathered together in this section are the lemmas which will be used in the proofs of the theorems. This section is concluded with a proof of Theorem 1 .

The following notation will be used. If $Q$ is any bounded, nondecreasing function over $(-\infty,+\infty)$, then $Q_{a c}, Q_{d}, Q_{c s}$ denote, respectively, the absolutely continuous, the discrete and the continuous singular components of $Q$. The bounded, nondecreasing functions $Q_{a c}, Q_{d}, Q_{c s}$ are uniquely determined if we 
set $Q(-\infty)=Q_{i}(-\infty)=0, i=a c, d, c s$. If $Q$ is a bounded, nondecreasing function, we shall let $\operatorname{Var} Q$ denote the total variation of $Q$. If $Q$ and $R$ are two bounded, nondecreasing functions, then $\operatorname{Var} Q * R=(\operatorname{Var} Q)(\operatorname{Var} R)$. We further denote $Q_{c}=Q_{a c}+Q_{c s}$.

In Lemma 1 we prove a somewhat stronger result than what we actually use.

LEMMA 1. If $X$ and $Y$ are (not necessarily independent) random variables, if $X$ is discrete, and if $G$ and $H$ denote the distributions of $X+Y$ and $Y$ respectively, then $\operatorname{Var} G_{i}=\operatorname{Var} H_{i}$, where $i=a c, c s, d$.

Proof. Let $\left\{x_{n}\right\}$ denote the values that $X$ takes with positive probabilities. Then

$$
P[X+Y \leqq z]=\sum_{n} P\left(\left[Y \leqq z-x_{n}\right] \mid\left[X=x_{n}\right]\right) P\left[X=x_{n}\right]
$$

As a function of $z$, the total variation of each component of $P\left(\left[Y \leqq z-x_{n}\right] \mid\left[X=x_{n}\right]\right)$, is the same as the total variation of the corresponding component of $P\left([Y \leqq z] \mid\left[X=x_{n}\right]\right)$. If we let $\operatorname{Var}_{i}$ denote the total variation of the $i$ th component, $i=a c, c s, d$, then we may write

$$
\begin{aligned}
\operatorname{Var}_{i} P[X+Y \leqq z] & =\sum_{n} \operatorname{Var}_{i} P\left(\left[Y \leqq z-x_{n}\right] \mid\left[X=x_{n}\right]\right) P\left[X=x_{n}\right] \\
& =\sum_{n} \operatorname{Var}_{i} P\left([Y \leqq z] \mid\left[X=x_{n}\right]\right) P\left[X=x_{n}\right] \\
& =\operatorname{Var}_{i} P[Y \leqq z],
\end{aligned}
$$

which proves the assertion.

LeMmA 2. If $F$ and $G$ are distribution functions, if $\operatorname{Var} F_{d}>0$, if $H=F * G$, and if $\operatorname{Var} G_{i}>0$, then $\operatorname{Var} H_{i}>0, i=a c, c s, d$.

Proof. We may write

$$
H=F_{d} * G_{d}+F_{d} * G_{c s}+F_{d} * G_{a c}+F_{c} * G .
$$

If $\operatorname{Var} G_{i}>0$, then $\operatorname{Var}\left(F_{d} * G_{i}\right)=\left(\operatorname{Var} F_{d}\right)\left(\operatorname{Var} G_{i}\right)>0$. By Lemma $1, F_{d} * G_{i}$ has property $i$, and thus $\operatorname{Var} H_{i} \geqq \operatorname{Var}\left(F_{d} * G_{i}\right)>0$, which proves the lemma.

Notation. If $E$ is a Lebesgue-measurable set of real numbers, we denote by $|E|$ the Lebesgue measure of $E$. Also, if $E_{1}, \cdots, E_{n}$ are $n$ sets of real numbers, we denote

$$
\begin{aligned}
E_{1} \oplus \cdots \oplus E_{n} & =\left\{x_{1}+\cdots+x_{n} \mid x_{i} \in E_{i}, 1 \leqq i \leqq n\right\}, \\
\bigoplus^{n} E_{1} & =\left\{x_{1}+\cdots+x_{n} \mid x_{i} \in E_{1}, 1 \leqq i \leqq n\right\} .
\end{aligned}
$$

LEMMA 3. There exists a perfect set $E \subset[0,1]$ such that $\left|\bigoplus^{n} E\right|=0$ for every $n$.

A proof of this lemma is found in [6, p. 103]. A construction of such a set, $E$, is as follows. Let $\left\{\xi_{n}\right\} \subset(0,1 / 2)$ be such that $\xi_{n}>\xi_{n+1}$, and $\xi_{n} \rightarrow 0$ as $n \rightarrow \infty$. Let $E_{1}=\left[0, \xi_{1}\right] \cup\left[1-\xi_{1}, 1\right]$, 


$$
E_{2}=\left[0, \xi_{1} \xi_{2}\right] \cup\left[\xi_{1}-\xi_{1} \xi_{2}, \xi_{1}\right] \cup\left[1-\xi_{1}, 1-\xi_{1}+\xi_{1} \xi_{2}\right] \cup\left[1-\xi_{1} \xi_{2}, 1\right],
$$

and, in general, $E_{n} \supset E_{n+1}, E_{n}$ is the disjoint union of $2^{n}$ closed intervals, each interval of length $\prod_{i=1}^{n} \xi_{i}$. Then one denotes $E=\bigcap_{n=1}^{\infty} E_{n}$.

LEMMA 4. In the construction of E given after Lemma 3,

(i) if $0<p_{i}<1,1 \leqq i \leqq n$, and if

$$
S_{n}=\left(E \cap\left[0, p_{1}\right]\right) \oplus \cdots \oplus\left(E \cap\left[0, p_{n}\right]\right),
$$

then $\left|S_{n}\right|=0$,

(ii) there exists a continuous distribution function $H$ such that $\int_{E} d H(x)=1$, and

(iii) for any $p \in(0,1]$ there is a continuous singular distribution function $H(\cdot \mid p)$ such that $\int_{E \cap[0, p]} d H(x \mid p)=1$.

Proof. (i) Since $S_{n} \subset \bigoplus^{n} E$, then $\left|S_{l i}\right|=0$.

(ii) $H$ is constructed in exactly the same way as the continuous singular distribution, whose support is the Cantor ternary set, is obtained.

(iii) Take $H$ as in (ii) and define

$$
H(x \mid p)= \begin{cases}H(x) / H(p) & \text { if } x \leqq p \\ 1 & \text { if } x>p .\end{cases}
$$

This concludes the proof of the lemma.

LEMMA 5. Let $\left\{Z_{n}\right\}$ and $\left\{Z_{n}^{*}\right\}$ be two sequences of random variables such that $\sum_{n} P\left[Z_{n} \neq Z_{n}^{*}\right]<1$. If $\sum_{n=1}^{\infty} Z_{n}^{*}$ converges a.s. (and therefore $\sum_{n=1}^{\infty} Z_{n}$ converges a.s.), and if the distribution function of $\sum_{n=1}^{\infty} Z_{n}^{*}$ is singular (i.e., it has no absolutely continuous component), then the distribution function of $\sum_{n=1}^{\infty} Z_{n}$ is not absolutely continuous.

Proof. Let $A_{n}=\left[Z_{n} \neq Z_{n}^{*}\right]$, and let $B=\bigcap_{n=1}^{\infty} A_{n}^{c}=\left[Z_{n}=Z_{n}^{*}\right.$ for all $\left.n\right]$. By hypothesis, $P(B)=1-P\left(\bigcup_{n=1}^{\infty} A_{n}\right)>0$. Since the distribution function of $\sum_{n=1}^{\infty} Z_{n}^{*}$ is singular there exists a set of real numbers, $L$, such that $|L|=0$ and $P\left[\sum_{n=1}^{\infty} Z_{n}^{*} \in L\right]=1$. Hence

$$
P\left[\sum_{n=1}^{\infty} Z_{n} \in L\right] \geqq P\left(B \cap\left[\sum_{n=1}^{\infty} Z_{n} \in L\right]\right)=P\left(B \cap\left[\sum_{n=1}^{\infty} Z_{n}^{*} \in L\right]\right)=P(B)>0,
$$

which implies that the distribution function of $\sum_{n=1}^{\infty} Z_{n}$ is not absolutely continuous.

LEMMA 6. Let E be a closed, bounded set of Lebesgue measure zero. Let

$$
E_{r}=\{x:|x-y| \leqq r \text { for some } y \in E\},
$$

where $r>0$. Then $\left|E_{r}\right| \rightarrow 0$ as $r \rightarrow 0$. 
Proof. Let $a<b$ and $E \subset[a, b]$. Then we may write $[a, b] \backslash E=\bigcup_{n=1}^{\infty}\left(a_{n}, b_{n}\right)$, where the intervals $\left\{\left(a_{n}, b_{n}\right)\right\}$ are disjoint, and the sequence $\left\{b_{n}-a_{n}\right\}$ is nonincreasing. Let $\varepsilon>0$. There exists an integer $N$ such that

$$
b-a-\sum_{n=1}^{N}\left(b_{n}-a_{n}\right)=\sum_{n=N+1}^{\infty}\left(b_{n}-a_{n}\right)<\varepsilon / 2
$$

(since $|E|=0$ ). Let $r_{0}>0$ be defined by $r_{0}<\varepsilon / 4 N$ (thus $r_{0}<\varepsilon / 4$ ) so that $2 N r_{0}<\varepsilon / 2$. Then for $r<r_{0}$ we have, using the notation $x^{+}=(x+|x|) / 2$,

$$
\begin{aligned}
\left|E_{r}\right| & =b-a-\sum_{n=1}^{\infty}\left(b_{n}-a_{n}-2 r\right)^{+} \\
& \leqq b-a-\sum_{n=1}^{N}\left(b_{n}-a_{n}-2 r\right)^{+} \\
& \leqq b-a-\sum_{n=1}^{N}\left(b_{n}-a_{n}\right)+2 N r<\varepsilon,
\end{aligned}
$$

which proves the lemma.

LEMMA 7. If $\left\{\phi_{n}(u)\right\}$ is a sequence of characteristic functions such that $\prod_{n=N}^{\infty} \phi_{n}(u)$ is the characteristic function of an absolutely continuous distribution function for every $N$, if $\left\{\lambda_{n}\right\}$ is a sequence of positive numbers such that $\sum_{n=1}^{\infty} e^{-\lambda_{n}}<\infty$, and if $\phi(u)=\exp \sum_{n=1}^{\infty} \lambda_{n}\left(\phi_{n}(u)-1\right)$ is a characteristic function, then $\phi(u)$ is the characteristic function of an (infinitely divisible) absolutely continuous distribution function.

Proof. Let $\left\{U_{n, k}, V_{n}, k, n=1,2, \cdots\right\}$ be independent random variables such that the characteristic function of $U_{n, k}$ is $\phi_{n}$ and the distribution of $V_{n}$ is Poisson with expectation $\lambda_{u}$. Then $\phi(u)$ is the characteristic function of

$$
Z=\sum_{n=1}^{\infty} \sum_{k=1}^{V_{n}} U_{n, k}
$$

which converges a.s. since it converges in distribution. Let

$$
\Omega_{n}=\left[V_{n}=0\right] \bigcap_{k=n+1}^{\infty}\left[V_{k}>0\right]
$$

for $n=1,2, \cdots$, and let $\Omega_{0}=\bigcap_{k=1}^{\infty}\left[V_{k}>0\right]$. The events $\left\{\Omega_{n}\right\}$ are disjoint. Since, by hypothesis, $\sum_{n=1}^{\infty} e^{-\lambda_{n}}<\infty$, then $P\left(\bigcup_{n=0}^{\infty} \Omega_{n}\right)=\sum_{n=0}^{\infty} P\left(\Omega_{n}\right)=1$. Let

$$
S_{n}=\sum_{k=n+1}^{\infty} U_{k, 1} \text { and } T_{n}=Z-S_{n} \text {. }
$$

The two events $\left[S_{n} \leqq s\right]$ and $\Omega_{n}$ are independent, and so are the two events: $\left[S_{n} \leqq s\right]$ and $\Omega_{n}\left[T_{n} \leqq t\right]$. (N. B. $\left[S_{n} \leqq s\right]$ and $\left[T_{n} \leqq t\right]$ are not independent, but 
when the latter event is replaced by its intersection with $\Omega_{n}$, then independence is obtained.) Hence

$$
\begin{aligned}
P\left(\left[S_{n} \leqq s\right]\left[T_{n} \leqq t\right] \mid \Omega_{n}\right) & =P\left(\left[S_{n} \leqq s\right]\left[T_{n} \leqq t\right] \Omega_{n}\right) / P\left(\Omega_{n}\right) \\
& =P\left[S_{n} \leqq s\right] P\left(\left[T_{n} \leqq t\right] \Omega_{n}\right) / P\left(\Omega_{n}\right) \\
& =P\left(\left[S_{n} \leqq s\right] \mid \Omega_{n}\right) P\left(\left[T_{n} \leqq t\right] \mid \Omega_{n}\right)
\end{aligned}
$$

Hence $S_{n}$ and $T_{n}$ are independent relative to the probability $P\left(\cdot \mid \Omega_{n}\right)$. But $P\left(\left[S_{n} \leqq s\right] \mid \Omega_{n}\right)=P\left[S_{n} \leqq s\right]$ is absolutely continuous by hypothesis for every $n$, and hence $P\left([Z \leqq z] \mid \Omega_{n}\right)$ is absolutely continuous since it is the convolution of two distributions, one of which is absolutely continuous. Since

$$
P[Z \leqq z]=\sum_{n=0}^{\infty} P\left([Z \leqq z] \mid \Omega_{n}\right) P\left(\Omega_{n}\right),
$$

then by Lemma 2 in [10], the distribution function of $Z$ is absolutely continuous.

Proof of Theorem 1. It was remarked in $\$ 1$ that condition (i) was proved sufficient for absolute continuity of $F$ in [2] and [10] and that each of the remaining conditions is obviously sufficient for absolute continuity of $F$. We need only prove here the necessity of at least one of the conditions. We first prove that if neither condition (i) nor condition (ii) holds, then $F^{(d)} * F^{(c s)}$ is absolutely continuous. If neither (i) nor (ii) is true, then $F^{(a c)}$, because of Lemma 3 in [10], is easily seen to be absolutely continuous over $\left(-\infty, x_{0}\right) \cup\left(x_{0}, \infty\right)$ and is discontinuous at $x_{0}=-f_{-\infty}^{\infty}(1 / x) d G_{a c}(x)$. Hence by Lemma $2, F^{(d)} * F^{(c s)}$ cannot have a discrete component, i.e., it is continuous. If $F^{(d)} * F^{(c s)}$ were continuous but not absolutely continuous, then, by Lemma $2, F=F^{(a c)} * F^{(c s)} * F^{(d)}$ would not be absolutely continuous, which is a contradiction, thus proving $F^{(d)} * F^{(c s)}$ absolutely continuous. Let us assume now that none of (i), (ii), (iii), (iv) are true and prove that $(v)$ is true. Since we have assumed that $F^{(d)}$ is not absolutely continuous, then by a theorem due to Hartman and Wintner [5], and referred to in $\S 1, F^{(d)}$ is discrete or continuous singular. We now prove that $F^{(d)}$ must be continuous singular. If it were not and were therefore discrete, then $F^{(d)} * F^{(c s)}$ will have a singular or discrete component, depending on which of these $F^{(c s)}$ has (because we have assumed (iv) is not true), because of Lemma 2. But we have just proved that if at least both (i) and (ii) are not true, then $F^{(d)} * F^{(c s)}$ is absolutely continuous. Thus $F^{(d)}$ is continuous singular. We now prove that $F^{(c s)}$ is continuous. If not, then it has a discrete component, from which we obtain that $F^{(c s)} * F^{(a c)}$ has a discrete component by Lemma 2 . Since $F^{(d)}$ is continuous singular, then, by Lemma $2, F=F^{(d)} * F^{(c s)} * F^{(a c)}$ has a continuous singular component, contradicting the hypothesis that $F$ is absolutely continuous. Hence $F^{(d)}$ is continuous singular, $F^{(c s)}$ is continuous but not absolutely continuous and $F^{(d)} * F^{(c s)}$ is absolutely continuous, which concludes the proof of Theorem 1. 
In order to show that this is a best enumerative theorem, we must show that it is possible for each of conditions (i)-(v) to hold. In order to show that condition (i) is possible, let $G_{a c}$ be defined by

$$
G_{a c}(x)= \begin{cases}0 & \text { if } x \leqq 0 \\ x & \text { if } 0<x<1, \\ 1 & \text { if } x \geqq 1\end{cases}
$$

and let $G=G_{a c}$. Condition (ii) is obviously possible. An example of condition (iii) holding is given in [5] and in [10]. The principal problem of this paper is to show that conditions (iv) and (v) are nonvacuous, and the remainder of this paper is devoted toward this objective.

3. The infinitely divisible distribution model of Herman Rubin. In this section a certain class of infinitely divisible distribution functions due to Herman Rubin, and considered by him in [9], is developed. Slight changes in his model are made here for purposes required in $\S 4$. Theorems 2 and 3 and the corollary to Theorem 3 are due to Rubin and may be found as Lemmas 1 and 2 and Corollary 1 in [9]. The proof given here of Theorem 2 is essentially that of Rubin, while the proof given here of Theorem 3 is somewhat different.

Let $\left\{U_{i j}, Y_{k}, i, j, k=1,2, \cdots\right\}$ be independent random variables, where for every $i$ and $j, P\left[U_{i j}=0\right]=P\left[U_{i j}=1\right]=1 / 2$, and the distribution of $Y_{k}$ is Poisson with expectation $\lambda_{k}>0$. We assume that

$$
\sum_{n=1}^{\infty} \lambda_{n}=\infty
$$

Let $\varepsilon_{n}>0$ be such that $\sum_{n=1}^{\infty} \varepsilon_{n}<\infty$. Let $\left\{b_{n}\right\}$ be an increasing sequence of positive integers, $\left\{c_{n}\right\}$ a nondecreasing sequence of positive integers, and $\left\{\sigma_{n}\right\}$ a sequence of positive real numbers, all of which are constructed as follows. (We do not use most of these requirements until §4.)

The nondecreasing sequence of positive integers $\left\{c_{n}\right\}$ is selected only in order that

$$
\sum_{n=1}^{\infty} P\left[Y_{n}>c_{n}\right]<1
$$

The increasing sequence of positive integers $\left\{b_{n}\right\}$ is selected to satisfy several requirements. The first one is that

$$
\sum_{n=1}^{\infty} c_{n} 2^{-b_{n}}<1
$$

Now let us denote

$$
r_{n}=\sum_{j=1}^{\infty} c_{n+j} 2^{-b_{n+j}}
$$


The second requirement on $\left\{b_{n}\right\}$ is that it increases fast enough so that

$$
r_{n}<\varepsilon_{n} 2^{-b_{n}}, \quad n=1,2, \cdots .
$$

(Note that this can be done since $r_{n}$ depends on $b_{n+1}, b_{n+2}, \cdots$.).

We assume that $\left\{\sigma_{n}\right\}$ decreases fast enough so that

$$
\sum_{n=1}^{\infty} \sigma_{n} c_{n}<\infty
$$

Now (3.5) permits the following notation:

$$
r_{n}^{\prime}=r_{n}+\sum_{j=1}^{\infty} \sigma_{n+j} c_{n+j} .
$$

Let $E$ denote a set constructed as illustrated after the statement of Lemma 3. Keeping $E$ fixed we denote

$$
\begin{aligned}
E_{m} & =E \cap\left[0, \sigma_{m}\right], \\
E_{m, n} & =E_{m} \oplus\left\{k / 2^{b_{n}}, 0 \leqq k \leqq 2^{b}\right\}, \\
E(n) & =E_{1, n} \oplus \cdots \oplus E_{n, n} .
\end{aligned}
$$

Note that $E(n)$ is a closed, bounded set, and by Lemma $4,|E(n)|=0$. Denote

$$
E(n)_{r_{n}^{\prime}}=\left\{x|x-y| \leqq r_{n}^{\prime} \text { for some } y \in E(n)\right\} .
$$

We require that $\left\{b_{n}\right\}$ increase fast enough and $\left\{\sigma_{n}\right\}$ decrease fast enough so that $\left\{r_{n}^{\prime}\right\}$ decreases to zero at a fast enough rate in order that, by Lemma 6 ,

$$
\left|E(n)_{r_{n}^{\prime}}\right|<\varepsilon_{n}, \quad n=1,2, \cdots .
$$

Let us denote

$$
\begin{gathered}
X_{i n}=\sum_{k=b_{n}+1}^{b_{n+1}} U_{i k} / 2^{k}, \quad Z_{n}=\sum_{i=1}^{Y_{n}} X_{i n}, \\
Z=\sum_{n=1}^{\infty} Z_{n}, \quad Y_{n}^{*}=\min \left\{Y_{n}, c_{n}\right\}, \\
Z_{n}^{*}=\sum_{i=1}^{Y_{n}^{*}} X_{i n}, \quad \text { and } Z^{*}=\sum_{n=1}^{\infty} Z_{n}^{*} .
\end{gathered}
$$

REMARK 1. For every $i$ and $n, X_{i n}$ is uniformly distributed over

$$
\left\{k / 2^{b_{n+1}}, 0 \leqq k \leqq 2^{b_{n+1}-b_{n}}-1\right\},
$$

and $\sum_{k=n}^{\infty} X_{1 k}$ is uniformly distributed over the interval $\left[0,2^{-b_{n}}\right]$ and is therefore absolutely continuous.

Proof of this remark is an easy computation. 
REMARK 2. The series $Z=\sum_{n=1}^{\infty} Z_{n}$ converges a.s.

Proof. Since $\left[Y_{n}^{*}=Y_{n}\right] \subset\left[Z_{n}^{*}=Z_{n}\right]$, then $\left[Z_{n}^{*} \neq Z_{n}\right] \subset\left[Y_{n}^{*} \neq Y_{n}\right]$, and we have by (3.2)

$$
\sum_{n=1}^{\infty} P\left[Z_{n}^{*} \neq Z_{n}\right] \leqq \sum_{n=1}^{\infty} P\left[Y_{n}^{*} \neq Y_{n}\right]=\sum_{n=1}^{\infty} P\left[Y_{n}>c_{n}\right]<1 .
$$

By the Borel-Cantelli lemma we need only prove that $\sum_{n=1}^{\infty} Z_{n}^{*}$ converges a.s. Since $0 \leqq X_{\text {ln }}<2^{-b_{n}}$ and $0 \leqq Z_{n}^{*}<c_{n} 2^{-b_{n}}$ a.s., we obtain that $\sum_{n=1}^{\infty} Z_{n}^{*}$ is monotone nondecreasing, uniformly bounded above (by (3.3)) by 1 , which proves the assertion.

REMARK 3. The distribution function of $Z$ is infinitely divisible, it is continuous, and its G-function is discrete.

Proof. The characteristic function of $Z$ is easily computed to be

$$
\exp \sum_{n=1}^{\infty}\left(\lambda_{n} / 2^{b_{n+1}-b}\right) \sum_{k=0}^{2^{b_{n+1}-b_{n}-1}}\left(e^{i u k / 2^{b_{n+1}}}-1\right)
$$

which is obviously infinitely divisible. The $M$-function (and therefore the $G$ function) is discrete, since, between $2^{-b_{n+1}}$ and $2^{-b_{n}}, M$ takes jumps of size $\lambda_{n} / 2^{b_{n+1}-b_{n}}$ at each of the points $\left\{k 2^{-b_{n+1}}, 0 \leqq k \leqq 2^{b_{n+1}-b_{n}}-1\right\}$ and is constant between consecutive jumps. Hence the sum of all these jumps is $\sum_{n=1}^{\infty} \lambda_{n}=\infty$, and thus by the theorem of Hartman and Wintner and by Blum and Rosenblatt mentioned in $\S 1$, the distribution function of $Z$ is continuous.

THEOREM 2. If $\sum_{n=1}^{\infty} e^{-\lambda_{n}}<\infty$, then the distribution function of $\mathrm{Z}$ is absolutely continuous.

Proof. If we denote the characteristic function of $X_{1 n}$ by $f_{n}(u)$ then the characteristic function of $Z$ is $\exp \sum_{n=1}^{\infty} \lambda_{n}\left(f_{n}(u)-1\right)$. Referring to Remark 1, this theorem is just a particular case of Lemma 7 .

THEOREM 3. If $\sum_{n=1}^{\infty} e^{-\lambda_{n}}=\infty$, then the distribution function of $Z$ is continuous singular.

Proof. Since the $G$-function of $Z$ is discrete, its distribution function, $F_{Z}$, is pure; this follows from a theorem due to Hartman and Wintner [5] mentioned in $\$ 1$. Hence, all that is needed is proof that $F_{Z}$ is continuous but not absolutely continuous. By Remark 3 we know that $F_{Z}$ is continuous. All that remains is to prove $F_{Z}$ is not absolutely continuous. By Lemma 5 , we need only prove that the distribution function of $Z^{*}$ is singular. For this we need only show that for every $\eta>0$ there is a random variable $Z_{\eta}$ and a measurable set of real numbers $L_{\eta}$ such that $Z_{\eta}=Z^{*}$ a.s., $\left|L_{\eta}\right|<\eta$ and $P\left[Z_{\eta} \in L_{\eta}\right]=P\left[Z^{*} \in L_{\eta}\right]=1$. For every positive integer $v$ we denote 


$$
\begin{aligned}
\eta_{v} & =\sum_{n=v}^{\infty} \varepsilon_{n}, \\
D_{v n} & =\left(\bigcap_{k=v}^{n-1}\left[Y_{k}^{*}>0\right]\right)\left[Y_{n}^{*}=0\right], \quad n=v, v+1, \cdots, \quad\left(D_{v v}=\left[Y_{n}^{*}=0\right]\right), \\
A_{v} & =\bigcup_{n=v}^{\infty} D_{v n}, \\
Z_{\eta v} & =Z^{*} I_{A v},
\end{aligned}
$$

and

$$
L_{\eta_{v}}=\bigcup_{n=v}^{\infty} \bigcup_{k=0}^{2_{n}}\left[k / 2^{b_{n}},\left(k / 2^{b}\right)+r_{n}\right] .
$$

The hypothesis and the Borel-Cantelli lemma imply that $P\left(A_{v}\right)=1$, and therefore $Z^{*}=Z_{\eta_{v}}$ a.s. for every $v$. For every fixed $v,\left\{D_{v n}, n=v, v+1, \cdots\right\}$ are disjoint, and

Now

$$
Z_{\eta_{v}}=\sum_{n=0}^{\infty} Z^{*} I_{D_{v}}
$$

$$
Z^{*} I_{D_{v . .}}=\left\{\left(Z_{1}^{*}+\cdots+Z_{n-1}^{*}\right)+\left(Z_{n+1}^{*}+\cdots\right)\right\} I_{D_{v n}} .
$$

But by (3.3),

$$
P\left[\left(Z_{1}^{*}+\cdots+Z_{n-1}^{*}\right) I_{D v n} \in\left\{k / 2^{b_{n}}, 0 \leqq k \leqq 2^{b_{n}}-1\right\}\right]=1,
$$

and by the definition of $r_{n}$ we have

$$
P\left[\left(Z_{n+1}^{*}+\cdots\right) I_{D v n} \in\left[0, r_{n}\right]\right]=1 .
$$

Let $S_{n}=\bigcup_{k=0}^{2^{b_{n}-1}}\left[k / 2^{b_{n}},\left(k / 2^{b_{n}}\right)+r_{n}\right]$. Then by (3.4), $\left|S_{n}\right| \leqq r_{n} 2^{b_{n}}<\varepsilon_{n}$. Since or each fixed $v$ the $D_{v n}$ 's are disjoint then it follows that

$$
\left[Z_{\eta_{v}} \in \bigcup_{n=v}^{\infty} S_{n}\right] \supset \bigcap_{n=v}^{\infty}\left[Z^{*} I_{D_{v_{n}}} \in S_{n}\right]
$$

or $P\left[Z_{\eta_{v}} \in L_{\eta_{v}}\right]=1$. But

$$
\left|\bigcup_{n=v}^{\infty} S_{n}\right| \leqq \sum_{n=v}^{\infty}\left|S_{n}\right|<\sum_{n=v}^{\infty} \varepsilon_{n},
$$

which becomes as small as we choose by taking $v$ sufficiently large, thus concluding the proof of the theorem.

COROllary to Theorem 3. There exists an infinitely divisible distribution function $F$ produced by a discrete $G$-function such that $F$ is continuous singular and $F * F$ is absolutely continuous. 
Proof. Take $\lambda_{n}=\log (n+1)$. Then $\lambda_{n}>0$, all $n, \sum_{n=1}^{\infty} \lambda_{n}=\infty$ and $\sum_{n=1}^{\infty} e^{-\lambda_{n}}=\infty$. Hence by Theorem 3, taking $F$ as the distribution function of $Z, F$ is continuous singular. By Remark 3 the characteristic function of $F * F$ is

$$
\exp \sum_{n=1}^{\infty}\left(2 \lambda_{n} / 2^{b_{n+1}-b_{n}}\right) \sum_{k=0}^{2^{b_{n}+1}-b_{n}-1}\left(e^{i u k / 2^{b_{n}+1}}-1\right),
$$

which is the characteristic function of $\sum_{n=1}^{\infty} \sum_{k=1}^{U_{n}} X_{k n}$, where $X_{k n}$ is as before, $U_{n}$ has a Poisson distribution with expectation $2 \lambda_{n}$, and $\left\{X_{k n}, U_{n}, k, n=1,2, \cdots\right\}$ are independent. Since $\sum_{n=1}^{\infty} e^{-2 \lambda_{n}}<\infty$, then by Theorem 2 we obtain that $F * F$ is absolutely continuous.

4. Nonvacuousness of conditions (iv) and (v). In this section we show that conditions (iv) and (v) of Theorem 1 can hold.

We let $U_{i n}, Y_{k}, X_{i n}, Z_{n}, Y_{k}^{*}, Z_{n}^{*}, Z^{*}$ and $Z$ remain as they were defined in $\S 3$, and we keep the same assumptions on $\left\{b_{n}\right\},\left\{c_{n}\right\}$ and $\left\{\sigma_{n}\right\}$. We consider in addition the random variables $\left\{U_{i m}^{\prime}, Y_{k}^{\prime}, W_{i m}, i, k, m=1,2, \cdots\right\}$ such that the distributions of $U_{i m}^{\prime}$ and $Y_{k}^{\prime}$ are the same as those of $U_{i m}$ and $Y_{k}$ respectively, such that the distribution function of $W_{i m}$ is $H\left(\cdot \mid \sigma_{m}\right), i=1,2, \cdots$. (which was defined in Lemma 4(iii)), and such that the set of random variables $\left\{U_{i m}, U_{i m}^{\prime}, W_{i m}, Y_{m}, Y_{m}^{\prime}, i, m=1,2, \cdots\right\}$ are all independent. The following notation is used:

$$
\begin{array}{rlrl}
X_{i n}^{\prime} & =\sum_{k=b_{n}+1}^{b_{n+1}} U_{i k}^{\prime} / 2^{k}, & Z_{n}^{\prime}=\sum_{i=1}^{Y_{n}^{\prime}} X_{i n}^{\prime}, \\
Z^{\prime}=\sum_{n=1}^{\infty} Z_{n}^{\prime}, & Y_{n}^{\prime *}=\min \left\{Y_{n}^{\prime}, c_{n}\right\}, \\
Z_{n}^{\prime *}=\sum_{i=1}^{Y^{*}} X_{i n}^{\prime}, & Z^{\prime *}=\sum_{n=1}^{\infty} Z_{n}^{\prime *}, \\
W^{\prime}=\sum_{n=1}^{\infty} \sum_{i=1}^{Y_{n}^{\prime}} W_{i n}, & W^{\prime *}=\sum_{n=1}^{\infty} \sum_{i=1}^{Y_{n}^{\prime *}} W_{i n} .
\end{array}
$$

Assumption (3.5) implies that $W^{\prime *}$ converges a.s., and hence by the definition of $\left\{c_{n}\right\}$ and by the Borel-Cantelli lemma, $W^{\prime}$ converges a.s.

THEOREM 4. The distribution function $F_{1}$ of the random variable $W^{\prime}+Z^{\prime}$ is infinitely divisible and has these properties: (i) $F_{1}$ is continuous but not absolutely continuous, and (ii) $F_{1}$ is produced by a continuous singular G-function.

Proof. We first prove infinite divisibility and (ii). By Lemma 1, the distribution function $H_{n}$ of $X_{i n}^{\prime}+W_{i n}$ is continuous singular, the characteristic function of $\sum_{n=1}^{Y_{n}^{\prime}}\left(X_{i n}^{\prime}+W_{i n}\right)$ is 


$$
\exp \lambda_{n} \int_{-\infty}^{\infty}\left(e^{i u x}-1\right) d H_{n}(x)=\exp \lambda_{n} \int_{-\infty}^{\infty}\left(e^{i u x}-1\right) d\left(H_{n}(x)-1\right),
$$

and the characteristic function of $W^{\prime}+Z^{\prime}$ is

$$
\exp \int_{-\infty}^{\infty}\left(e^{i u x}-1\right) d H(x)
$$

where $H(x)=\sum_{n=1}^{\infty} \lambda_{n}\left(H_{n}(x)-1\right)$ for $x>0$ and $H(x)=0$ for $x \leqq 0$. Thus $F_{1}$ is infinitely divisible and the $M$-function, $H(x)$, which produces it is continuous singular, thus verifying (ii). In order to prove (i) we first prove that the distribution of $W^{*}+Z^{*}$ is singular. We observe that

$$
\begin{aligned}
& 0 \leqq X_{i n}^{\prime} \leqq 2^{-b_{n}} \quad \text { a.s., } \\
& 0 \leqq X_{i n}^{\prime}+W_{i n} \leqq 2^{-b_{n}}+\sigma_{n} \text { a.s., }
\end{aligned}
$$

and

$$
0 \leqq \sum_{i=0}^{\mathbf{Y}_{n}^{\prime *}}\left(X_{i n}^{\prime}+W_{i n}\right) \leqq c_{n}\left(2^{-b_{n}}+\sigma_{n}\right) \text { a.s. }
$$

By the definition of $r_{n}^{\prime}$ we have

$$
P\left[\sum_{n=N+1}^{\infty} \sum_{j=1}^{Y_{n}^{\prime *}}\left(X_{j n}^{\prime}+W_{j n}\right) \in\left[0, r_{N}^{\prime}\right]\right]=1
$$

Let $A_{v}$ and $D_{v n}$ be as in the proof of Theorem 3 but defined in terms of $Y_{n}^{\prime *}$ instead of $Y_{n}^{*}$. Then by the definition of $E(n)_{r_{n}^{\prime}}$ given in $\S 3$,

$$
P\left[\left(W^{\prime *}+Z^{\prime *}\right) I_{D_{v n}} \in E(n)_{r_{n}^{\prime}}\right]=1 .
$$

Since for fixed $v,\left\{D_{v n}\right\}$ are disjoint, we have

$$
P\left[\left(W^{\prime *}+Z^{*}\right) I_{A_{v}} \in \bigcup_{n=v}^{\infty} E(n)_{r_{n}^{\prime}}\right]=1
$$

for every v. By (3.6),

$$
\left|\bigcup_{n=v}^{\infty} E(n)_{r_{n}^{\prime}}\right|<\sum_{n=v}^{\infty} \varepsilon_{n},
$$

which can be made a small as we please by taking $v$ sufficiently large. Thus $W^{\prime *}+Z^{\prime *}$ has a singular distribution. By Lemma 5 , the distribution function of $Z^{\prime}+W^{\prime}$ is not absolutely continuous. Continuity of the distribution function of $Z^{\prime}+W^{\prime}$ follows from the fact that $\sum_{n=1}^{\infty} \lambda_{n}=\infty$, which concludes the proof of the theorem. 
THEOREM 5. Let $\left\{\lambda_{n}\right\}$ satisfy (3.1) and be such that

$$
\sum e^{-\lambda_{n}}=\infty \text { and } \sum e^{-2 \lambda_{n}}<\infty .
$$

Then the distribution function of $Z+Z^{\prime}+W^{\prime}$ is absolutely continuous.

Proof. Even though the distribution of $Z+Z^{\prime}$ is easily proved to be absolutely continuous by the corollary to Theorem $3, W^{\prime}$ is not independent of $Z+Z^{\prime}$, at least not apparently so, since both are defined in terms of $\left\{Y_{n}^{\prime}\right\}$; hence the conclusion of the theorem cannot be drawn so easily. Let $f_{n}$ and $g_{n}$ be the characteristic functions of $X_{1 n}$ and $W_{1 n}$ respectively. Then the characteristic function of $Z+Z^{\prime}+W^{\prime}$ is

$$
\begin{aligned}
\psi(u) & =\exp \left\{\sum_{n=1}^{\infty} \lambda_{n}\left(f_{n}(u)-1\right)\right\} \exp \left\{\sum_{n=1}^{\infty} \lambda_{n}\left(f_{n}(u) g_{n}(u)-1\right)\right\} \\
& =\exp \sum_{n=1}^{\infty} 2 \lambda_{n}\left(f_{n}(u) \frac{\left(1+g_{n}(u)\right)}{2}-1\right) .
\end{aligned}
$$

In order to apply Lemma 7 we need only verify that

$$
\phi_{N}(u)=\prod_{n=N}^{\infty} f_{n}(u) \frac{1+g_{n}(u)}{2}
$$

is the characteristic function of an absolutely continuous distribution function for every $N$. Now $\left(1+g_{n}(u)\right) / 2$ does happen to be the characteristic function of the distribution function

$$
F_{n}(x)= \begin{cases}0 & \text { if } x<0, \\ \left(1+H\left(x \mid \sigma_{n}\right)\right) / 2 & \text { if } x \geqq 0 .\end{cases}
$$

Let $W_{1 n}^{\prime}$ be a random variable with distribution function $F_{n}(x)$. We construct $\left\{W_{1 n}^{\prime}\right\}$ so that the random variables $\left\{X_{1 n}^{\prime}, W_{1 n}^{\prime}\right\}$ are independent. Thus $\phi_{N}(u)$ is the characteristic function of

$$
\sum_{n=N}^{\infty}\left(X_{1 n}+W_{1 n}^{\prime}\right)
$$

which is unconditionally convergent (in Loève's terminology in [7, p. 538]) and therefore converges to the same limit random variable under all reorderings and partitioned summations (ibid., p. 539). Hence

$$
\phi_{N}(u)=\left(\prod_{n=N}^{\infty} f_{n}(u)\right)\left(\prod_{n=N}^{\infty} \frac{1+g_{n}(u)}{2}\right) .
$$

But by Remark $1, \prod_{n=N}^{\infty} f_{n}(u)$ is the characteristic function of an absolutely continuous distribution function, and hence so is $\phi_{N}(u)$. The conclusion of the theorem follows from Lemma 7.

Theorem 5 proves that condition (v) in Theorem 1 is nonvacuous. This follows from the fact that $Z$ and $Z^{\prime}+W^{\prime}$ are independent. 
It is now easy to establish that condition (iv) of Theorem 1 is also possible. To do this, let $\left\{\lambda_{n}\right\}$ satisfy $\sum_{n=1}^{\infty} e^{-\lambda_{n}}<\infty$. We need to show that under this hypothesis, $Z^{\prime}+W^{\prime}$ is absolutely continuous. In this case the characteristic function of $Z^{\prime}+W^{\prime}$ is $\exp \left\{\sum_{n=1}^{\infty} \lambda_{n}\left(f_{n}(u) g_{n}(u)-1\right)\right\}$. As in the proof of Theorem 5 , for every $N$

$$
\prod_{n=N}^{\infty}\left(f_{n}(u) g_{n}(u)\right)=\left(\prod_{n=N}^{\infty} f_{n}(u)\right)\left(\prod_{n=N}^{\infty} g_{n}(u)\right),
$$

each of the infinite products on the right is a characteristic function, and the first is (by Remark 1) the characteristic function of an absolutely continuous distribution function for every $N$. By Lemma 7 , the distribution of $Z^{\prime}+W^{\prime}$ is absolutely continuous.

5. Acknowledgments. I wish to express my gratitude to Professor Herman Rubin who showed me his construction of $Z$ and informed me of the results contained in Theorems 2 and 3 and the corollary to Theorem 3 at the 1963 Summer Meeting of the American Mathematical Society at Boulder, Colorado. Especial thanks go to Professor J.-P. Kahane for a most enjoyable and fruitful collaboration in considering the problem of the nonvacuousness of condition ( $v$ ) of Theorem 1.

\section{REFERENCES}

1. J. R. Blum and M. Rosenblatt, On the structure of infinitely divisible distributions, Pacific J. Math 9 (1959), 1-7.

2. M. Fisz, Infinitely divisible distributions: recent results and applications, Ann. Math. Statist. 33 (1962), 68-84.

3. M. Fisz and V. S. Varadarajan, A condition for absolute continuity of infinitely divisible distribution functions, Z. Wahrscheinlichkeitstheorie und Verw. Gebiete 1 (1963), 335-339.

4. B. V. Gnedenko and A. N. Kolmogorov, Limit distributions for sums of independent random variables, Addison-Wesley, Cambridge, Mass., 1954.

5. P. Hartman and A. Wintner, On the infinitesimal generators of integral convolutions, Amer. J. Math. 64 (1942), 273-298.

6. J.-P. Kahane and R. Salem, Ensembles parfaits et séries trigonométriques, Actualités Sci. Indust. No. 1301, Hermann, Paris, 1963.

7. M. Loève, Probability theory, 2nd ed., Van Nostrand, Princeton, N.J., 1960.

8. E. Lukacs, Characteristic functions, Hafner, New York, 1960.

9. H. Rubin, Supports of infinitely divisible distributions, Tech. Rep. No. RM-108 HR-10, Michigan State Univ., Department of Statistics, East Lansing, Mich., 1963.

10. H. G. Tucker, Absolute continuity of infinitely divisible distributions, Pacific J. Math. 12 (1962), 1125-1129.

11. - On continuous singular infinitely divisible distribution functions, Ann. Math. Statist. 35 (1964), 330-335.

UNIVERSITY OF CALIFORNIA,

Riverside, CALIFoRNia

THE INSTITUTE FOR AdVANCEd StUdy,

Princeton, New Jersey 